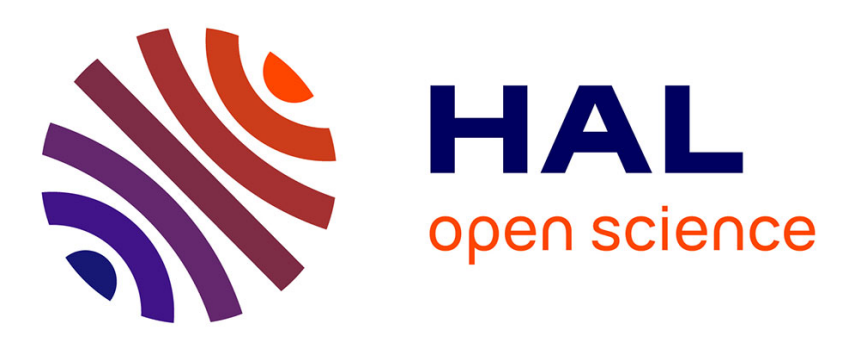

\title{
Sur les interactions entre la robotique et les sciences de l'esprit et du comportement
}

\author{
Pierre-Yves Oudeyer
}

\section{To cite this version:}

Pierre-Yves Oudeyer. Sur les interactions entre la robotique et les sciences de l'esprit et du comportement. eds. C. Garbay et D. Kaiser. Informatique et Sciences Cognitives : influences ou confluences?, Presses Universitaires de France, 2009. inria-00420309

\section{HAL Id: inria-00420309 https://hal.inria.fr/inria-00420309}

Submitted on 28 Sep 2009

HAL is a multi-disciplinary open access archive for the deposit and dissemination of scientific research documents, whether they are published or not. The documents may come from teaching and research institutions in France or abroad, or from public or private research centers.
L'archive ouverte pluridisciplinaire HAL, est destinée au dépôt et à la diffusion de documents scientifiques de niveau recherche, publiés ou non, émanant des établissements d'enseignement et de recherche français ou étrangers, des laboratoires publics ou privés. 


\title{
Sur les interactions entre la robotique et les sciences de l'esprit et du comportement
}

\author{
Pierre-Yves Oudeyer \\ INRIA Bordeaux-Sud Ouest \\ http://www.pyoudeyer.com
}

C'est en 1912 que les ingénieurs John Hammond Jr. et Benjamin Miessner construisirent l'un des premiers robots, qui fit grand bruit dans la presse américaine et secoua le monde scientifique (Cordeschi, 2002). Ce robot, appelé «Electric dog», était une machine électrique à roues dont le mécanisme la faisait s'orienter vers les sources lumineuses et les suivre selon des trajectoires à la fois réactives et complexes. A l'instar du magazine «Electrical Experimenter », la presse et le grand public décrirent très vite cette machine comme «pensante», "d'une intelligence presque surhumaine», et comme l'une des « inventions les plus sensationnelles » qui aient été faites. L'impact fut encore accru par le fait que Hammond et Miessner destinaient cette technologie à un usage militaire : ils voulaient équiper torpilles et missiles de ce système, afin qu'ils puissent automatiquement viser les batteries anti-aériennes ennemies qui de nuit utilisaient de puissants projecteurs lumineux (Miessner, 1912).

En réalité, le mécanisme sous-jacent était relativement simple : pour le mettre au point, Hammond et Miessner s'étaient inspirés des travaux du grand biologiste Jacques Loeb et de ses théories sur le comportement phototropique d'un certain nombre d'insectes, et en particulier les papillons de nuit. Alors que d'une part le grand débat du vitalisme faisait encore rage, avec les défenseurs de l'idée qu'un «principe vital» non matériel était nécessaire à l'explication du comportement des êtres vivants (Driesch, 1909; McDougall, 1911), et que d'autre part des scientifiques comme Alfred Binet, Francis Darwin ou Ernest Haeckel utilisaient un langage anthropomorphique pour décrire le comportement des animaux les plus simples en leur attribuant par exemple "volonté 》 ou " conscience " (Cordeschi, 2002), Jacques Loeb proposait qu'au moins un certain nombre de comportements pouvaient être expliqués par des mécanismes réflexes purement chimiques et mécaniques. En particulier, il défendit l'idée que le comportement des insectes phototropiques, qui se manifestent par des trajectoires apparemment complexes autour des sources lumineuses, pouvaient être expliqué simplement par le fait que les muscles du côté de l'animal qui est frappé par la lumière devenaient plus actifs que ceux du côte opposé. Loeb proposait ainsi de considérer ces insectes comme de pures «machines chimiques héliotropiques ». Cette théorie fut présentée dans son livre « Comparative Physiology of the Brain and Comparative Psychology » en 1900 (Loeb, 1900). Cependant, ses arguments verbaux ne convinquirent pas et reçurent les critiques des vitalistes et de ceux qui pensaient qu'un système plus compliqué était nécessaire pour reproduire le comportement de ces insectes.

C'est ainsi que Loeb découvrit avec grand intérêt la machine construite par Hammond et Miessner. Ils avaient réussi à construire de manière totalement mécanique, par un couplage électromécanique direct entre des capteurs de lumière et des moteurs reprenant les principes énoncés par Loeb, une "machine artificielle héliotropique» dont le comportement reproduisait avec succès celui des insectes phototropes. Le "electric dog " venait ainsi prouver la cohérence et la plausibilité de ses théories, que la communauté scientifique ne lui avait pas accordée auparavant. Dans un nouveau livre qui cette fois eut un impact beaucoup 
plus grand (Forced Movements, Tropisms, and Animal Conduct, 1918), Loeb reprit en détail la description du robot et écrit :

« [Il me semble que] la construction effective d'une machine héliotrope ne supporte pas seulement les conceptions mécanistiques des actions volontaires et instinctives des animaux mais aussi [ma] théorie de l'héliotropisme, puisque cette théorie a servi de base à la construction de cette machine. » (Loeb, 1918 :69).

Le robot de Hammond et Miessner ne démontrait pas seulement la plausibilité et la cohérence de la théorie de Loeb, il portait également un coup important aux vitalistes et à ceux qui proposaient d'attribuer "volonté », " conscience » ou " téléologie » aux animaux les plus simples. Tout d'abord, il était une preuve flagrante que l'on pouvait reproduire le comportement organisé et héliotrope d'un être vivant uniquement avec des mécanismes physiques: le principe vital ne semble donc pas être nécessaire pour expliquer certains comportements spécifiques du vivant. Ensuite, il montrait que l'on pouvait générer un comportement apparemment volontaire et téléologique, mais dont l'inspection des mécanismes internes révélait qu'ils étaient beaucoup plus simples. Ceci fut magnifié par toutes les réactions et interprétations qu'en firent la presse et le grand public, quand ils parlèrent de machine « d'une intelligence presque surhumaine ».

Ce dernier point d'ailleurs identifie une autre contribution importante de l' " electric dog », qu'il partage avec ses successeurs, comme l'homéostat de Ashby (Ashby, 1940) qui fut qualifié de " machine pensante ", la tortue électrique de Grey Walter qui fit sensation au festival d'Angleterre en 1951 (Walter, 1951), le joueur de dames d'Arthur Samuel qui obtint des audiences très fortes sur la chaine CBS en 1960 (Samuel, 1959), ou plus récemment les robots footballeurs de la Robocup (http://www.robocup.org/): la présence de ces robots permet de mettre explicitement en lumière certains des a priori cognitifs des humains, et en particulier leur tendance à anthropomorphiser, quand ils tentent d'expliquer les phénomènes qu'ils observent, comme le comportement des animaux. L' " electric dog » fut donc, même si c'est un effet collatéral, un outil permettant d'étudier non pas seulement la plausibilité des théories de Loeb ou du vitalisme, mais aussi l'observateur humain lui-même, qu'il soit scientifique ou pas.

L'histoire de l' " electric dog », bien qu'elle fut finalement encore un cas isolé en ce début de $20^{\text {ème }}$ siècle dans lequel le terme « robot » n'existait pas encore, est cependant emblématique des relations que la robotique et les sciences du vivant, en particulier les sciences du l'esprit et du comportement, a entretenu par la suite, et dans les dernières décennies en particulier (Cordeschi, 2002). Elle démontre d'abord l'apport que les sciences du vivant peuvent faire à la technologie, et à la robotique en particulier. C'est probablement l'aspect le plus connu des interactions entre ces domaines (Arkin, 1998). Cependant, l'autre versant de cette interaction est tout aussi important et c'est sur quoi ce chapitre va se concentrer : on y décèle la manière dont la construction de robots peut avoir un impact profond et varié sur la manière dont les scientifiques conçoivent le vivant et la pensée. Des hypothèses importantes peuvent être pour la première fois mises à l'épreuve expérimentale, soit en en prouvant leur cohérence interne, soit en en prouvant leur non-nécessité ou leur suffisance. En outre, par cette construction, ces hypothèses sont conduites à être formulées plus complètement, plus explicitement et plus opérationnellement. De nouvelles hypothèses peuvent aussi être le résultat de cette construction. Parallèlement, grâce à cette méthode expérimentale et l'usage de l'artificiel, l'épistémologie et l'activité même du théoricien se trouvent questionnées. Le robot n'est pas seulement utilisé pour modéliser, mais aussi comme dispositif expérimental pour étudier les 
humains (ou les animaux) qui interagissent avec lui. Nous allons maintenant revenir sur des exemples plus récents, en montrant à chaque fois comment ils illustrent l'un de ces types particuliers de contribution de la robotique aux sciences de l'esprit et du comportement.

\section{Les robots, modèles opérationnels pour mettre des hypothèses spécifiques à l'épreuve}

Alors que l'impact de l' « electric dog » sur les sciences du vivant fut un effet collatéral des travaux de Hammond et Miessner, dont l'objectif était avant tout de s'inspirer des travaux de Loeb pour construire des machines plus efficaces, les deux dernières décennies du $20^{\text {ème }}$ siècle ont vu le développement de tout un ensemble de projets de robotique dont l'objectif explicite était de mettre à l'épreuve des hypothèses proposées par des biologistes (Webb, 2000). De manière intéressante, on retrouve notamment un grand nombre de modèles robotiques du comportement des mêmes insectes qui passionnaient Loeb. Soixante quinze ans plus tard, on ne se disputait plus sur la présence ou non d'un principe vital, mais sur les mécanismes physiques et chimiques exacts qui sont mis en œuvre chez ces insectes (Webb, 2000).

Un défi important est par exemple la compréhension des mécanismes qui permettent aux insectes de voler en ligne droite, de se fixer sur un objectif, de suivre un objectif ou tout simplement d'atterrir (Srinivasan et al., 1999, Huber et Bulthoff, 1998, Franceschini et al., 2007). En effet, les insectes ont la particularité d'avoir des yeux immobiles et à focale fixe, ce qui les empêche de déterminer la distance des objets qu'ils perçoivent au travers de la vergence binoculaire ou de l'effort réfractif qu'il faut faire pour les voir net. Un certain nombre de biologistes ont alors proposé l'hypothèse que les insectes utilisaient l'information de mouvement de l'image, techniquement appelée flux optique (Exner, 1891; Wallace, 1959). Plusieurs indices calculés à partir du mouvement des images, comme la différence des vitesses globales perçues dans chaque œil, ont été ensuite proposés et testés d'abord dans des expériences avec des mouches ou des abeilles (Srinivasan et al., 1991 ; Srinivasan et al., 1999). Bien que contrôlées et encourageantes, elles ne permettaient pas de conclure que ces indices de flux optique seuls pouvaient permettre d'expliquer la navigation visuelle des abeilles. En outre, certains biologistes avaient proposé que ces indices étaient utilisés par des circuits réflexes neuronaux très simples, mais la plausibilité restait spéculative. Des robots ont alors été construits, dans lesquels étaient implémenté le calcul de ces indices de mouvement de l'image, couplé à des systèmes de contrôle réflexe très simple (Srinivasan et al., 1999, Santos-Victor et al., 1995). On a alors montré que cela permettait effectivement à un robot de naviguer par exemple au centre de couloirs ou de tunnels de largeurs variables et de directions changeantes de la même manière que les abeilles (Santos-Victor et al., 1995). Cependant, on s'est aperçu que l'utilisation de mécanismes réflexes posait parfois problèmes dans des environnements contenant des patchs de surfaces non-texturées, et qu'il était nécessaire d'ajouter un dispositif plus complexe, impliquant une forme de mémoire à court terme, pour rendre la navigation aussi robuste que celle des abeilles. Ces expériences robotiques montraient ainsi que le flux optique seul était la source d'une information suffisante pour pouvoir naviguer, mais que des mécanismes purement réflexes ne permettaient pas d'expliquer seuls la robustesse des comportements de navigation chez les abeilles et les mouches.

Plus spécifique encore, d'autres chercheurs ont utilisé des robots pour étudier la validité d'hypothèses physiologiques sur les circuits neuronaux qui implémentent les mécanismes de contrôle des comportements de navigation des mouches. Des biologistes ont proposés que la même structure neuronale simple était à la fois à l'origine des comportements de stabilisation de trajectoires, de vol stationnaire, et d'approche d'objets stationnaires (Götz and Wenking, 
1973), et identifiés un ensemble de neurones potentiellement impliqués dans ce circuit (Egelhaaf et al., 1989). Sur cette base, ce circuit a alors été implémenté sur un robot, et on a montré qu'effectivement il permettait de générer de manière robuste la variété des comportements en question (Huber et Bulthoff, 1998).

Un autre exemple de la manière dont les robots ont pu permettre l'évaluation et l'élaboration d'hypothèses comportementales est celui de projet RoboTuna (Triantafyllou et Triantafyllou, 1995), qui s'intéresse aux mécanismes de la nage chez les poissons et les dauphins. Les performances de nage des poissons et des dauphins sont en effet paradoxales : en 1936, le zoologiste James Gray calcula la force musculaire nécessaire à un dauphin pour atteindre une vitesse de 20 nœuds, comme cela est parfois observé, et en prenant en compte la résistance de l'eau contre le corps du dauphin. La comparaison de ce résultat avec les modèles musculaires du dauphin établis par les biologistes se révéla étonnante : le dauphin était sept fois trop faible ! Une première hypothèse proposa que les modèles musculaires des dauphins étaient faux, et qu'en réalité leurs muscles devaient être bien plus efficaces que ceux des mammifères terrestres. Cependant, certains se demandèrent si la solution de ce paradoxe n'était pas du côté de l'hydrodynamique. En effet, outre leur vitesse phénoménale, les dauphins et les poissons sont aussi capables de changer totalement de direction sans perte de vitesse et en faisant des virages très étroits, ce que sont incapables de faire tous les engins nautiques (bateaux, sousmarins, ...) conçus par l'homme et basé sur les connaissances hydrodynamiques classiques, aussi puissants soient-ils. L'hypothèse des « vortex » fut alors avancée : alors qu'ils nagent, les poissons et les dauphins pourraient créer des turbulences hydrodynamiques nonconventionnelles dont ils se serviraient pour diminuer leur trainée et augmenter leur puissance. Soixante ans après l'énonciation du paradoxe de Gray, la controverse n'était toujours pas résolue, les connaissances en biologie et en théorie hydrodynamique n'étant toujours pas assez avancées. C'est dans ce contexte que le projet Robotuna vit le jour (voir http://web.mit.edu/towtank/www/Tuna). Une équipe d'ingénieur construisit ainsi un robot dont la morphologie était calquée sur celle d'un thon, équipé d'un ensemble de moteurs classiques dont on connaissait précisément la puissance, et qui permettaient de produire des oscillations tout le long du corps du robot, du nez jusqu'à la queue, afin qu'il puisse nager en ligne droite dans un bassin d'essai. Les paramètres de ces oscillations furent alors optimisés expérimentalement afin de maximiser la vitesse du robot. On compara alors cette vitesse maximale avec celle prédite par le même calcul que celui que James Gray avait utilisé pour le dauphin, et basée d'une part sur la connaissance parfaite de la forme et des moteurs du robot, et d'autre part sur les modèles hydrodynamiques conventionnels : la vitesse expérimentale du robot thon était nettement supérieure à celle de la vitesse prédite (mais encore inférieure à celle d'un vrai thon). Le paradoxe de Gray était donc reproduit, et l'expérience rendit l'hypothèse des vortex beaucoup plus plausible, tout en suggérant fortement que la différence des muscles marins/terrestres n'était probablement pas la réponse.

\section{Les robots, preuves de concepts et outils d'exploration}

Si de nombreux robots ont été développés pour étudier, comme dans les exemples précédents, des hypothèses relativement spécifiques sur le comportement d'animaux simples (pour un panorama plus large, voir les actes des conférences Simulation of Adaptive Behavior, http://www.isab.org, ou Webb, 2000), un nombre tout aussi important d'autres expériences robotiques ont été mises en œuvre pour explorer et valider des hypothèses plus génériques, et en particulier concernant le rôle des relations cerveau-corps-environnement ou l'explication de phénomènes cognitifs de plus haut niveau comme l'imitation ou le langage. C'est ce que nous allons voir maintenant. 
L'inscription corporelle de l'intelligence et du comportement. Pendant la majeure partie du $20^{\text {ème }}$ siècle, l'intelligence des organismes de haut niveau comme les humains a été conçue avant tout comme un système de traitement de l'information et de manipulation de symbole au travers de règles logiques d'inférence, et dans lequel le corps n'est qu'une interface qui en entrée transforme les perceptions en symboles et exécute en sortie les ordres calculés par le système d'inférence (Chomsky, 1957; Newell et Simon, 1976 ; Putnam, 1975; Dupuy, 2005). C'est ce qu'on appelle le cognitivisme, qui fut à la fois au cœur de l'intelligence artificielle et des sciences cognitives (McCarthy et al., 1955 ; Boden, 2006). Cette approche fut plébiscitée dans les années 1950-1970, et renforcée par les premiers résultats de l'intelligence artificielle qui par exemple réussit à construire des programmes capables de jouer très bien aux dames (Samuel, 1959) ou à prouver automatiquement des théorèmes (Ernst et Newell, 1969) - activités que le cognitivisme tenait comme caractérisant l'essence de l'intelligence. Cependant, les premières failles apparurent en même temps que les limites de ces programmes d'intelligence artificielle: les programmes experts en échec étaient incapables d'apprendre à jouer à un autre jeu, et les prouveurs de théorèmes incapables par exemple de comprendre les blagues les plus simples (Dreyfus, 1972). L'idée que le cognitivisme passait peut être à côté de propriétés fondamentales de l'intelligence et du comportement commença à faire son chemin.

Une première idée fut d'avancer que plutôt que de résider dans le cerveau et dans le système de symboles et d'inférences, l'intelligence et le comportement étaient le résultat des interactions dynamiques entre le cerveau, le corps et l'environnement. On proposait donc que le corps n'était pas seulement une interface, mais bien partie intégrante des mécanismes de l'intelligence. Proposée et argumentée par un certain nombre de philosophes (e.g. MerleauPonty, 1942, 1962; Dreyfus and Dreyfus, 1982), cette hypothèse ne commença à faire une réelle percée dans les sciences cognitives qu'à la suite d'un certain nombre d'expériences robotiques qui l'articulaient et l'illustraient explicitement. Nous allons en donner deux exemples emblématiques.

L'expérience des Didabots (Maris et te Boeckhorst, 1996) permet de montrer explicitement l'impact de la morphologie, en particulier les propriétés physiques et topologiques du corps, de ses moteurs et de ses senseurs, sur le comportement en comparant des robots dont le contrôleur est le même mais le corps différent. Les Didabots sont des robots à roues dotés de deux capteurs infra-rouge permettant de détecter la présence d'un obstacle potentiel dans une zone de cinq centimètres. Deux versions des Didabots ont été construites, qui différent par l'emplacement de ces deux capteurs (voir Figure 1): dans la version (A) les deux capteurs sont disposés sur le «nez » du robot, alors que dans la version (B), les capteurs sont disposés sur les « joues ». A ces capteurs, un même contrôleur très simples est associé à dans les deux cas, et qui correspond à un mécanisme d'évitement d'obstacle : si le capteur de droite est activé, alors tourner sur la gauche; si le capteur de gauche est activé, alors tourner sur la droite. On retrouve en fait un contrôleur quasiment identique à celui de 1' "electric dog ", mais inversé. On dispose alors les deux types de Didabots dans deux arènes identiques, qu'on a parsemé au hasard de cubes légers. Pour les robots de morphologie (A), on observe ce à quoi on pouvait s'attendre : ils évitent les obstacles. Les robots (B) évitent aussi les obstacles, mais un phénomène nouveau se produit: au bout d'un certain temps, on observe un regroupement massif des cubes qui avaient été disposés dans l'arène (voir Figure 2). Les robots (B) ont donc produit un comportement qu'un observateur extérieur qualifie typiquement en disant qu'ils ont « rangé » ou «nettoyé » l'areine. En fait, cela s'explique par le fait que si un robot de type (B) s'approche d'un cube de pleine face, il ne le détecte pas comme un obstacle étant donné que ses capteurs sont légèrement orientés sur les côtés (voir 
Figure 1). Il le pousse alors, jusqu'à ce qu'un de ses capteurs (droit ou gauche) perçoive un obstacle, typiquement un autre cube, et se mette à tourner. Le résultat est que deux cubes initialement non contigus se retrouvent posés l'un à côté de l'autre. De proche en proche, tous les cubes se trouvent rassemblés en quelques grands groupes dans l'arène. En conclusion, cette expérience montre clairement que le comportement des robots (B) n'est ni le simple fait de son cerveau/contrôleur, ni le simple fait de son corps (qui sans cerveau ne bougerait pas), mais de l'interaction entre les deux. Bien que ce type d'expérience soit relativement simple, elle a une importance scientifique très importante, car elle permet de faire ce qui n'est pas possible avec des animaux ou des humains : comparer des comportements à corps variable et contrôleur/cerveau constant (et vice versa). En effet, même pour une espèce donnée, du fait de la variabilité naturelle entre les individus, deux individus n'ont jamais exactement ni le même corps ni le même cerveau. Il n'y a donc pas de constante de référence. Plus encore, il est encore plus problématique par exemple d'imaginer pouvoir faire une expérience dans laquelle par exemple on ferait contrôler le corps d'un lion et d'un serpent par le même cerveau. Ce type d'expérience permettrait de faire avancer fondamentalement notre compréhension des rapports entre corps et cerveau dans le comportement. C'est ainsi que l'on comprend, étant donné qu'il n'est pas possible de les réaliser avec des animaux, l'impact des expériences robotiques dans lesquelles pour la première fois on peut considérer le corps comme une variable (Kaplan et Oudeyer, à paraître).

(A)

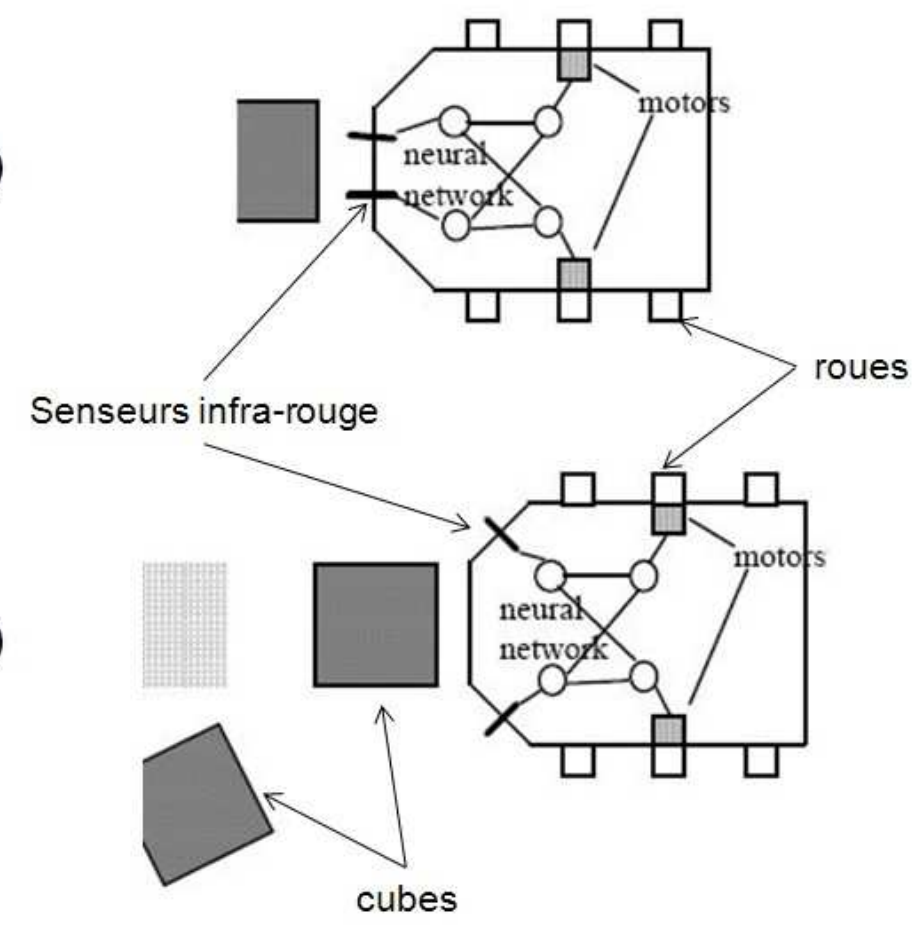

Figure 1 Morphologie des deux types de Didabots (Adapté de Pfeifer, 2000) 

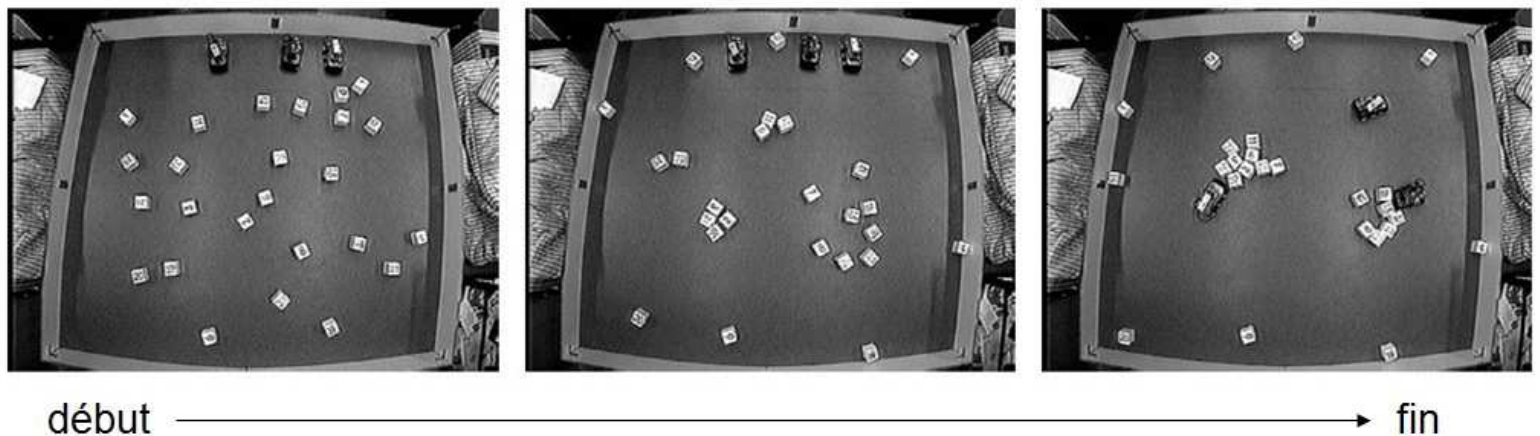

début

fin

Figure 2 Avec la morphologie (B) et le même controlleur, les Didabots rassemblent les cubes en tas (Adapté de Maris et te Boeckhorst, 1996)

L'idée sous-jacente à l'expérience des Didabots, et qui a été développée de manière systématique par Rolf Pfeifer et ses collègues (Pfeifer et Scheier, 1999), est que de manière implicite le corps lui-même, par ses propriétés physico-dynamiques, peut réaliser un «calcul» qui participe tout autant au comportement que le calcul explicite réalisé par le contrôleur/cerveau. C'est ainsi que pour une tâche donnée, une morphologie adaptée peut permettre de simplifier radicalement la complexité du contrôleur. Une expérience robotique extrème qui permet d'illustrer cette idée est celle réalisée par McGeer sur les robots marcheurs dynamiques passifs ( Passive dynamic walkers », McGeer, 1990, 1993, Coleman et al., 1998). La marche bipède est en effet un comportement complexe, mettant en jeu la coordination dynamique d'un grand nombre de muscles et de parties des jambes, pour laquelle certains ont proposé des systèmes de contrôle moteur d'une grande complexité, en particulier pour maintenir le corps en équilibre face à l'inertie du corps, aux frictions entre ses composants, et à la gravité (voir par exemple le système de contrôle de la marche du robot humanoïde ASIMO, Hirose et al., 2001). En complète opposition avec cette vision, McGeer, suivi d'autres chercheurs, a mis au point un dispositif entièrement mécanique reproduisant certains aspects de la morphologie des jambes humaines (voir Figure 3) mais totalement dénué de contrôleur (le dispositif était donc uniquement constitué de pièces de métal et de bois). Il a montré qu'avec des rapports adequats de poids, de taille et de position entre les différentes parties de ce " robot », et quand on le plaçait sur une surface légèrement inclinée, celui-ci se mettait à la descendre en marchant tout en gardant son équilibre et de manière étonnement ressemblante à la démarche humaine. Il avait donc construit un robot qui utilisait la gravité et les propriétés mécanique dynamique de son corps pour se mouvoir et garder l'équilibre, sans le moindre cerveau (et d'ailleurs aussi sans source interne d'énergie). Par la suite, des études systématiques ont montré comment l'ajout de contrôleurs et d'actionneurs simples pouvait permettre de construire un robot capable de marcher sur un terrain plat, avec un coût énergétique limité. Ce robot montrait donc de manière encore plus nette le rôle que peut avoir la morphologie dans la réalisation d'un comportement de coordination sensorimotrice complexe. 


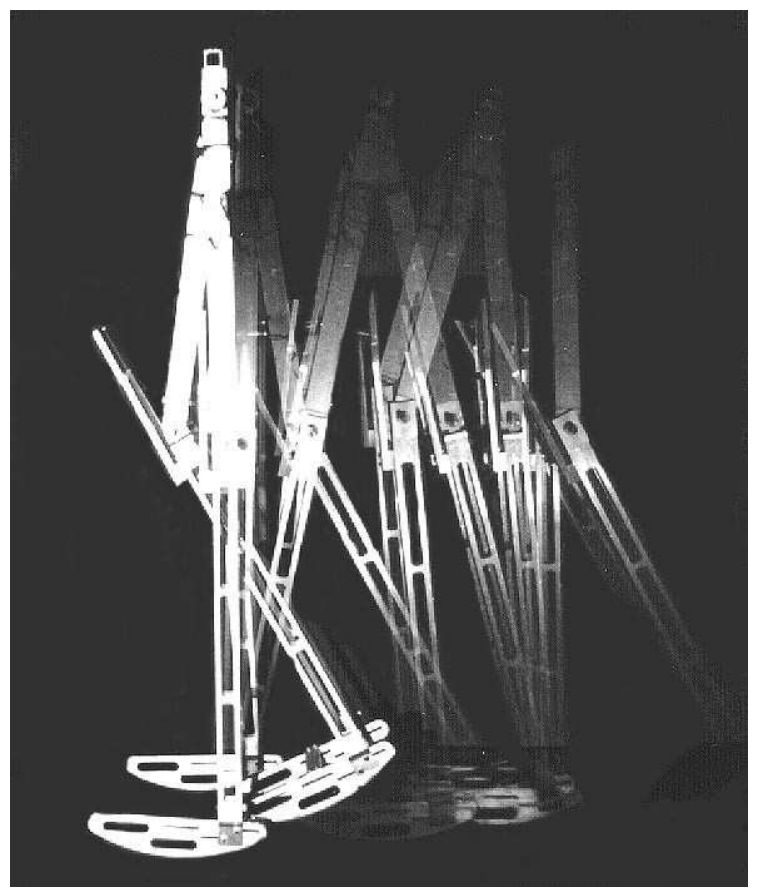

Figure 3 Un robot marcheur dynamique passif (Photo reprise de: http://ruina.tam.cornell.edu/hplab/pdw.html)

L'intelligence sans représentation. L'un des points centraux du cognitivisme est la notion de représentation, modèle interne que le cerveau se fait du monde et à partir duquel il doit pouvoir raisonner afin d'en déduire les actions qui vont lui permettre d'atteindre ses objectifs, et sans lequel un comportement de haut niveau ne doit pas être possible (McCarthy et al., 1955 ; Newell et Simon, 1976). Toute une série d'expériences en robotique sont venu contredire cette nécessité (Brooks, 1991). Si l'on a montré que des robots mobiles étaient capables d'apprendre à réaliser un certain nombre de tâches dans un environnement inconnu, comme par exemple retourner à un point de référence ou passer l'aspirateur sans utiliser de cartes de ce cet environnement et uniquement grâce à des mécanismes réflexes simples (McKenzie et Balch, 1993 ; Arkin, 1992), des expériences plus marquantes ont été réalisées à propos de capacité cognitive comme l'imitation. Très tôt les bébés humains commencent à imiter leurs congénères, et d'aucuns l'ont pris comme une preuve qu'ils devaient avoir un système avancé de représentations corporelles et sociales (Guillaume, 1925). En effet, l'imitation consistant par exemple à reproduire un geste observé, semble impliquer la capacité à se représenter la différence entre soi et l'autre (le geste observé n'est pas le sien) et se représenter les correspondances entre son propre espace moteur et celui de l'autre pour être capable de prendre sa perspective. Or, une expérience en robotique présentée dans (Andry et al., 2001) montre que cette conclusion ne peut pas être faite. Dans cette expérience, le système moteur du robot consiste en un bras, et son système sensoriel en une caméra sensible aux mouvements dans l'image. Le robot dispose d'un système d'apprentissage qui lui permet d'apprendre à prédire les mouvements visuels qu'il perçoit, typiquement provoqués par son bras qui passe dans son champ de vision, en fonction de ses commandes motrices. Le robot est également doté d'un système de motivation implémentant le principe d'homéostasie cognitive (von Foerster, 1958 ; Maturana et Varela, 1980) : quand le robot perçoit une erreur entre ce qu'il prédit et ce qu'il perçoit visuellement en réalité, il agit de manière à rétablir l'accord entre ses positions/mouvements moteurs, perçues de manière proprioceptive, et ses 
perceptions visuelles. (Andry et al., 2001) ont alors montré que si l'on mettait en face de ce robot un second robot avec la même morphologie et qu'on le faisait bouger, alors le premier se mettait à l'imiter spontanément. La perception des mouvements du bras du second robot provoquait en effet une disparité entre ce que le premier prédisait à partir de ses propres mouvements. Pour faire disparaître cette erreur, le premier robot s'est donc mis à produire le même mouvement, couvrant et suivant visuellement celui de l'autre robot. Du point de vue d'un observateur extérieur, leur robot imitait les gestes de celui qui était en face. Cependant, la connaissance parfaite de son sytème de contrôle permettait d'affirmer qu'il ne disposait d'aucune représentation de la différence entre ce qui relevait de son propre corps et ce qui n'en relevait pas (le corps d'un autre par exemple). A fortiori, le robot n'avait pas non plus de représentation de son alter ego, et aucun mécanisme de prise de perspective n'était mis en œuvre. Cette expérience ne montre évidemment pas que c'est ce mécanisme qui est à l'œuvre chez les très jeunes enfants, et par ailleurs de nombreux arguments convaincants indiquent que les formes d'imitation complexe qu'ils montrent quand ils sont plus grands nécessitent une représentation de soi et une compréhension de l'intentionnalité de l'autre (Tomasello et Carpenter, 2007). Par contre, cette expérience prouve que l'on ne peut pas déduire, à partir de l'observation des formes primitives d'imitation, que cela implique une représentation de soi, de l'autre et des correspondances entre les deux.

Auto-organisation et langage. L'auto-organisation est un concept issu des sciences de la complexité qui a eu et continue d'avoir de profondes conséquences sur la manière dont les scientifiques pensent la matière, le vivant et l'esprit. L'auto-organisation caractérise les systèmes dont les propriétés macroscopiques résultent de celles de leurs constituants tout en en étant qualitativement différentes. Elle caractérise par exemple la formation spontanée des cristaux de glace dans certaines conditions de température et de pression, avec des branches à la fois complexes et parfaitement symétriques dont la structure n'apparaît pas à l'échelle des molécules d'eau qui les composent (voir Figure 4). Après quelques précurseurs comme D’Arcy Thompson (Thompson, 1917) qui étudièrent des phénomènes auto-organisés sans les nommer, le concept est réellement apparu et s'est développé sous l'impulsion de chercheurs comme William Ross Ashby, Heinz von Foerster, Ilya Prigogine, Francesco Varela et René Thom. Il est maintenant au centre de la compréhension de nombreux phénomènes à la fois en sciences physique ou en biologie (Ball, 2001) : c'est le cas par exemple pour la formation des cristaux de glace, des distributions des avalanches dans les tas de sables ou dans les montagnes, des dunes dans le désert, des formes des deltas fluviaux, des galaxies ou des polyèdres de bulles au pied des cascades. C'est le cas aussi par exemple pour la formation des zébrures ou des tâches sur les corps des animaux, des motifs en spirales ou en étoiles dans les colonies de bactéries, pour la construction des nids de termites ou des fourmilières ou la formation de structure sociales dynamiques pour la chasse ou la récolte de sources d'aliments chez les abeilles.

En outre, ces phénomènes d'auto-organisation sont souvent compliqués à comprendre ou à prévoir intuitivement, et à formuler verbalement. Les outils mathématiques existant ne permettent pas non plus d'aller très loin dans de nombreux cas. L'une des manières les plus efficaces pour développer notre compréhension de la dynamique des systèmes auto-organisés est l'utilisation d'ordinateurs et de simulations. En effet, ils permettent d'élaborer des modèles dont on connaît toutes les hypothèses, de les faire fonctionner, et d'en observer le comportement selon les valeurs des paramètres fixés dans le cadre de ces modèles.

Ces modèles computationnels ont ainsi été au cœur du travail des physiciens et des biologistes travaillant sur les systèmes complexes depuis les années 1950, à commencer par les travaux 
de Alan Turing sur la morphogenèse (Turing, 1952) ou ceux de Enrico Fermi sur les dynamiques non-linéaires des interactions atomiques. Dans les années 1980, ils ont été utilisés intensément par les éthologues et ont permis de faire de grandes avancées sur la compréhension des sociétés d'insectes (Camazine et al., 2001).

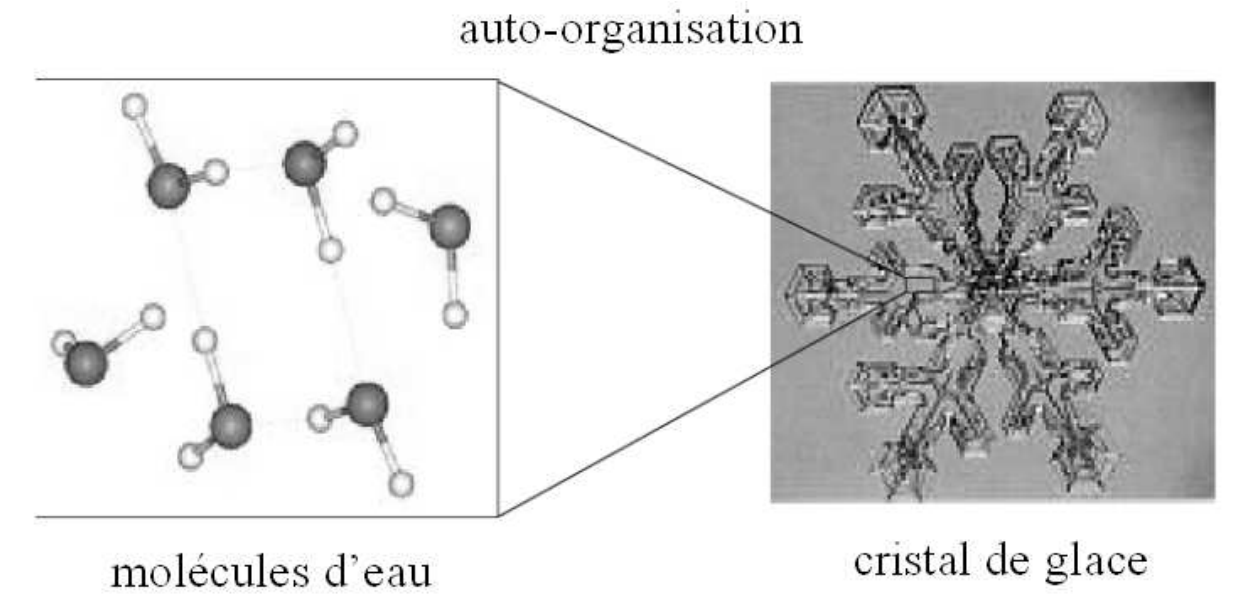

Figure 4 Le phénomène d'auto-organisation. Les propriétés globales du cristal de glace sont qualitativement différentes des propriétés locales des molécules d'eau

Plus récemment, un certain nombre de chercheurs ont proposé que l'auto-organisation, non seulement pouvait caractériser les propriétés de systèmes physiques ou biologiques " simples ", mais pouvait intervenir de manière tout aussi fondamentale dans les mécanismes cognitifs et sociaux qui caractérisent l'homme, par exemple concernant le langage et ses origines (Hurford et al., 1998). Ainsi, dans les deux dernières décennies du 20ème siècle, d'aucuns ont proposé qu'un certain nombre de structures linguistiques, plutôt que d'être codées dans le génome de manière explicite et innée (Pinker et Bloom, 1990), pourraient être le résultat de l'interaction dynamique et complexe de modules cérébraux génériques, de l'appareil vocal-gestuel-auditif-visuel des individus, des individus entre eux et de la fonction de communication (Lindblom et al., 1984 ; Hurford, 2001 ; Steels, 2003 ; Oudeyer, 2006). Plus loin encore, ils ont proposé que les langues elles-mêmes, systèmes conventionnels partagés par tous les individus d'une même communauté linguistique mais dont les mécanismes de formation sont restés longtemps très mystérieux, pouvaient être conceptualisées comme des macro-structures auto-organisées résultant des interactions culturelles locales entre individus (Steels, 1995, 2003). Cependant, ces hypothèses étaient audacieuses à deux points de vue : 1) elles s'opposaient frontalement à la linguistique Chomskienne et innéiste qui dominait depuis les années $1950^{1}$;2) les phénomènes auto-

\footnotetext{
${ }^{1}$ A noter que ce qu'on appelle ici « linguistique Chomskienne » est le courant de la linguistique structuraliste et innéiste, proposant que le cerveau des humains possède des structures neuronales innées spécifiques au langage et en particulier possédant une connaissance a priori des structures syntaxiques des langues humaines, et dont la présence est le résultat des mécanismes de l'évolution génétique sous une pression de sélection pour la communication linguistique. Aujourd'hui, le représentant emblématique de ce courant est Steven Pinker. Au contraire, Noam Chomsky est aujourd'hui plutôt un opposant de cette approche, restant le plus souvent neutre sur la manière dont les structures syntaxiques sont représentées et codées dans le cerveau et sur la question de savoir quelle est l'origine de ces codages, ou proposant parfois que l'origine de ses structures
} 
organisés sont complexes et souvent non-intuitifs, rendant toute théorie verbale s'y référant très spéculative. C'est pourquoi très vite, à l'instar des simulations informatiques des sociétés d'insectes sociaux en éthologie, des expériences robotiques furent mises au point pour mettre ces hypothèses à l'épreuve de la plausibilité et de la cohérence. L'utilisation de robots était ici essentielle, car les hypothèses basées sur l'auto-organisation mettaient en jeu les interactions entre le cerveau, l'espace moteur et perceptuel, et l'environnement physique et social. Nous allons ainsi présenter maintenant une expérience permettant d'illustrer ce que peuvent apporter les robots à ce problème fondamental de sciences humaines.

L'origine des systèmes de vocalisations et de leurs universaux. Bien qu'ils aient un appareil phonatoire et perceptuel qui leur permet de produire plusieurs centaines de voyelles et de consonnes, les humains n'utilisent le plus souvent qu'entre cinq et dix voyelles et à peine quelques dizaines de consonnes dans une langue donnée (Schwartz et al., 1997). En outre, chaque langue à son propre système de voyelles et de consonnes, produisant une forte diversité, mais en même temps, certaines voyelles et consonnes sont beaucoup plus fréquentes que d'autres, faisant ressortir des régularités statistiques structurelles (Schwartz et al., 1997). Plusieurs explications réductionnistes ont été proposées, qu'elles soient morphologiques (e.g. Stevens, 1972) ou génétiques (e.g. Mehler et al., 2000), mais elles ne permettaient pas de comprendre la dualité diversité/régularités statistiques que l'on observe. Certains ont alors proposé que les systèmes sonores des êtres humains étaient des compromis plus ou moins optimaux entre distinctivité acoustique et effort articulatoire et ont mis au point des simulations informatiques permettant de montrer qu'effectivement les systèmes optimaux étaient ceux qui étaient les plus fréquents dans les langues humaines (Liljencrantz et Lindblom, 1972). Cependant, ces simulations mettaient en œuvre des mécanismes d'optimisation explicites, dont on ne savait pas comment ils pouvaient être réalisés dans la nature, et ne répondaient pas à la question de savoir comment une communauté d'individus pouvait « choisir » collectivement un système plutôt qu'un autre.

pourrait être un effet collatéral, potentiellement auto-organisé, d'évolutions biologiques indépendantes de la communication. En ce sens, Chomsky n'est pas (ou plus) Chomskien. 


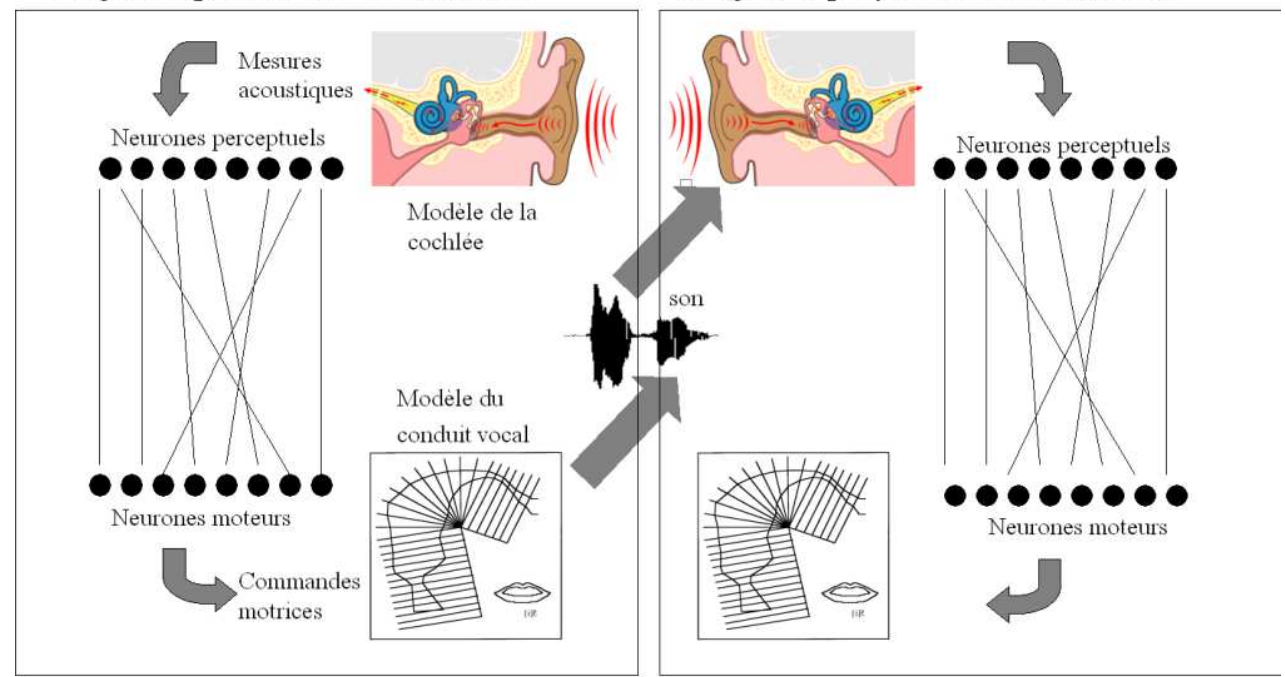

Figure 5 L'architecture des robots dans les expériences sur les origines des systèmes de vocalisations présentées dans (Oudeyer, 2006). Les agents sont dotés d'un oreille artificielle, d'un conduit vocal artificiel, et d'un cerveau artificiel qui couple ces deux organes. Les agents sont eux-mêmes couplés par l'environnement commun dans lequel ils évoluent : ils perçoivent les vocalisations de leurs voisins.

Des éléments de réponses ont alors pu être apportés par les robots construits et présentés successivement dans (de Boer, 2001) et (Oudeyer, 2005a, 2005b, 2006). Dans ces expériences, des robots simulés dans des mondes virtuels, étaient dotés de modèles physiques du conduit vocal, de l'oreille et de circuits neuronaux connectant les deux (voir Figure 5). Ces robots interagissaient deux par deux et localement dans l'espace, au travers d'un jeu d'imitation : dans une interaction, l'un des deux robots choisissait un son de son répertoire, le produisait, puis l'autre tentait de l'imiter en reproduisant le son de son répertoire le plus proche. Le premier agent vérifiait alors si le son imité était bien plus similaire à celui qu'il avait produit qu'à un autre son de son répertoire. Il faisait alors savoir au second agent si l'imitation avait été un succès ou un échec. Dans tous les cas, les deux agents mettaient à jour leurs répertoires de sons en faisant des hypothèses pour tenter de maximiser le nombre d'imitations réussies dans le futur. Les sons des répertoires des agents consistaient en des associations entre des trajectoires motrices et des trajectoires acoustiques, auxquelles étaient associés des scores. Le score d'une association augmentait si elle était utilisée dans une imitation réussie, et diminuait dans le cas contraire. Au départ, les agents commençaient avec un répertoire vide, qui augmentait à la fois par inventions aléatoires et par apprentissage lors des interactions. Le système de scores associés aux sons dans les répertoires, couplé au jeu de l'imitation, introduisait ainsi une dynamique Darwinienne culturelle, dans laquelle des sons et des groupes de sons pouvaient rentrer en compétition ou en coopération pour se « « reproduire» d'individus à individus (Oudeyer et Kaplan, 2007). Les expériences ont alors montré que très rapidement s'auto-organisait dans chaque société de robots un système de vocalisations partagé par tous les membres d'une même communauté, et que ce système était différent d'une communauté à l'autre. Cela a ainsi permis de montrer comment il était possible qu'une société d'individus sans coordination centrale pouvait converger vers un système de vocalisations conventionnalisé. En outre, dans ces expériences, on a montré que par exemple les systèmes de voyelles qui étaient formés par les sociétés de robots étaient à la fois divers et caractérisés par des régularités statistiques: certains systèmes de voyelles 
apparaissaient plus fréquemment que d'autres, tout en laissant la possibilité que de temps en temps des systèmes rares se forment. Plus encore, les systèmes de voyelles les plus fréquents apparaissant dans ces populations de robots étaient les mêmes que ceux apparaissant le plus fréquemment dans les langues humaines. Ces simulations montraient donc de manière convaincante la plausibilité et la cohérence de l'hypothèse de l'auto-organisation: des mécanismes simples, par leurs interactions dynamiques non-linéaires, pouvaient donner naissance à des systèmes sonores dotés des caractéristiques fondamentales des systèmes de vocalisations humains.

Origine du lexique, de la syntaxe, et apprenabilité. Aux côtés des expériences robotiques présentées dans les paragraphes précédents, toute une famille d'autres expériences est venue ces dernières années renforcer l'hypothèse de l'auto-organisation pour comprendre les origines de langues. Par exemple l'expérience des Tètes Parlantes (Kaplan, 2001 ; Steels et Kaplan, 2002; Steels, 2003) a montré comment une société de robots pouvait construire un lexique partagé, uniquement au travers d'interactions sociales locales. Cela a permis de montrer que c'était possible sans supposer de systèmes de catégories sémantiques innées, comme cela est proposé par les défenseurs de l'innéisme (Pinker et Bloom, 1990). D'autres expériences concernent l'origine des conventions syntaxiques et grammaticales (e.g. Kirby, 2001 ; Steels, 2005), et ont également montré comment un système linguistique pouvait évoluer culturellement pour s'adapter aux biais cognitifs génériques des individus (e.g. Zuidema 2003 ; Oudeyer et Kaplan, 2007). Dans ce cadre, ils ont montré que les systèmes linguistiques pouvait être sélectionnés pour leur apprenabilité : certaines structures avaient évolué pour être plus facilement apprenables par les individus, et en fonctions des contraintes génériques de leur cerveau. Ces expériences ont ainsi permis de contredire les conclusions qui avaient été faites par les innéistes d'après l'observation que les enfants apprennent leur langue maternelle si vite et avec si peu d'indices : ils proposaient que leur cerveau avait du s'adapter pour encoder des contraintes spécifiques à la langue afin de pouvoir l'apprendre aussi facilement. $\mathrm{Au}$ contraire, les expériences robotiques ont montré qu'il était tout à fait envisageable que ce soit les langues qui se soient adaptées à des cerveaux génériques.

\section{Les robots, dispositifs expérimentaux scientifiques et thérapeutiques pour la cognition animale et humaine.}

$\mathrm{Au}$ début du $20^{\mathrm{ème}}$ siècle, le robot de Hammond et Miessner, puis quelques années plus tard les machines d'Ashby et Walter, avaient indirectement donné l'occasion de mettre en lumière la manière dont les humains pouvaient sur-interpréter le comportement d'entités très simples. Bref, ils furent non seulement une occasion de faire avancer le débat opposant vitalistes et matérialistes, mais aussi d'étudier la psychologie humaine. Dans la dernière décennie du 20 ème siècle, certains chercheurs ont commencé à employer volontairement les robots comme dispositifs d'expérimentation et d'étude directe du comportement animal et humain, et non pas comme modèles de ces comportements comme dans les travaux que nous avons présentés dans les sections précédentes. Cependant, un point commun avec ces travaux de modélisation est l'utilisation des robots dans le cadre d'expériences s'intéressant à un large spectre de comportements : nous allons donner l'exemple de l'étude de certains comportements des insectes sociaux, puis de mammifères comme les chiens, et enfin de l'homme.

La danse des abeilles. Un exemple paradigmatique de l'utilisation de robots comme dispositif expérimental pour l'étude directe du comportement animal est celui présenté par (Michelsen et al., 1992) sur la danse des abeilles. Karl von Frisch a défendu la théorie selon 
laquelle les abeilles utilisaient leur danse de manière symbolique et partiellement arbitraire pour désigner à leurs congénères l'emplacement de sources de nectar (von Frisch, 1971). Selon cette théorie, quand une abeille revient à la ruche pour indiquer une source de nectar, deux cas se présentent. Si cette source est située relativement proche (moins de cent mètres), l'abeille opère une danse circulaire. Les autres abeilles viennent la toucher de leurs antennes pour sentir l'odeur du nectar encore collé sur les poils de la danseuse. Elles partent alors à la recherche de la source, guidées uniquement par leur odorat. Au contraire, si la source de nectar est relativement éloignée, la "danse frétillante» est effectuée. Elle consiste en la description successive de deux demi-cercles, en passant par une ligne droite centrale sur laquelle l'abeille agite son abdomen et émet un bourdonnement avec ses ailes. Von Frisch a proposé que la vitesse du frétillement et la fréquence du bourdonnement, ainsi que la période nécessaire pour effectuer un demi-cercle, déterminaient la distance pour aller à la source de nectar, tandis que l'angle formé entre la ligne centrale et le demi-cercle déterminait la direction de la source du nectar par rapport au soleil. Malgré l'obtention du prix Nobel en 1973, la théorie de von Frisch fut vivement contestée par plusieurs chercheurs qui proposaient que même dans le cas où la source de nectar était éloignée, c'était l'odorat des abeilles qui les guidait essentiellement (Wenner and Wells, 1990). Cette controverse était basée sur le fait que d'une part les abeilles disposent effectivement d'un odorat très développé, et d'autre part sur la relative incertitude du rôle des différentes composantes de la danse dans la théorie de von Frisch. En effet, à cause de la variabilité intrinsèque à chaque danse et chaque abeille, et à cause du fait que l'abeille produit toujours en même temps un ensemble complexe de signaux qu'il est difficile de déméler et dont on est pas sûr de les avoir tous identifiés, la théorie de von Frisch est relativement difficile à valider expérimentalement et de manière certaine.

C'est en 1992 qu'une expérience basée sur l'utilisation d'un robot est venue finalement la confirmer. Michelsen et ses collègues ont mis au point un petit robot de la taille et de la forme d'une abeille, relié à un ordinateur et téléguidé par un programme informatique, auquel ils ont pu faire effectuer des danses dans des ruches afin d'étudier leur impact sur ses congénères biologiques. Ce robot a d'abord été enduit de cire et placé un certain temps dans la ruche afin de s'imprégner de l'odeur des abeilles pour qu'il soit reconnu comme l'une des leurs. Une fois la phase d'habituation terminée, les chercheurs ont pu demander à leur robot d'effectuer un certain nombre de danses, dans lesquelles pouvait être contrôlés indépendamment la vitesse du frétillement, la fréquence du bourdonnement (grâce aux ondes acoustiques de petites ailes artificielles), la période pour effectuer les demi-cercles et l'angle entre la ligne droite centrale et les demi-cercles. L'immense avantage d'utiliser un robot était d'une part la possibilité de répéter exactement la même danse de nombreuses fois sans variabilité de manière à pouvoir étudier statistiquement la réponse comportementale des abeilles, et d'autre part de pouvoir par exemple enlever certaines composantes de la danse, les contrôler une à une, et même proposer des danses avec des composantes contradictoires qui ne sont jamais générées naturellement par les abeilles mais qui permettent de mettre en lumière la manière dont sont utilisés conjointement les différents indices. En outre, on pouvait demander au robot de faire une danse indiquant aux abeilles qu'il y avait une source de nectar à un endroit où il n'y en avait pas : cela permettait d'éliminer complètement la possibilité qu'elles se rendent à cet endroit en étant guidées par leur odorat. Les résultats de ces expériences furent très clairs : le robot des expérimentateurs arrivait à " envoyer» les abeilles où les expérimentateurs le voulaient, et sans indices olfactifs. Comme par ailleurs l'utilisation d'un robot permettait de dire que les seuls signaux communiqués aux abeilles étaient ceux implémentés dans le robot et correspondant à la théorie de von Frisch, les grandes lignes de celle-ci s'en trouvèrent confirmées. Mais l'utilisation du robot permis d'aller plus loin encore et de raffiner la théorie 
de von Frisch : Michelsen et ses collègues ont montré qu'en fait, la partie essentielle de la danse est celle de la ligne droite verticale pendant laquelle l'abeille/le robot agite son abdomen et émet un bourdonnement, alors que la partie demi-cercle, pendant laquelle l'abeille s'arrête de frétiller et de bourdonner pour revenir au point de départ de la ligne droite, ne semble pas avoir le rôle crucial d'indication de la direction que von Frisch avait proposé. Au contraire, il semble que ce soit la combinaison du son et du frétillement qui indique à la fois la distance et la direction de la source de nectar. On voit donc ici le rôle fondamental que le robot a pu jouer dans la compréhension de la danse des abeilles.

La reconnaissance de conspécifiques chez les chiens. Un autre exemple de l'utilisation de robots comme dispositifs expérimentaux est celui présenté dans (Kubinyi et al., 2004) et concernant l'étude de la reconnaissance des conspécifiques, c'est-à-dire des animaux de la même espèce, chez les chiens. L'étude des mécanismes de reconnaissance sociale, et en particulier de reconnaissance des animaux de la même espèce, est un sujet important en éthologie (Colgan, 1983). Pour de nombreuses espèces, les éthologues se posent d'une part la question de savoir si la reconnaissance de conspécifiques est innée ou apprise, comment elle se développe, si elle dépend du contexte, mais aussi quels sont les indices visuels, comportementaux, acoustiques où encore olfactifs qui permettent aux animaux de se reconnaître. Or, le problème est le même que pour les abeilles : dans la nature, les stimuli auxquels sont confrontés les animaux sont toujours fortement multidimensionnels et multimodaux, mélangeant un grand nombre d'indices potentiels que les expérimentateurs ne peuvent contrôler. Pour contourner ce problème, certains ont essayé d'utiliser des images générées artificiellement et systématiquement pour étudier la réaction potentielle des animaux, comme pour les poissons (Colgan, 1983), où ont mis au point des poupées/maquettes dont ils pouvaient contrôler l'apparence visuelle et les odeurs (Lack, 1939). Cependant, il a été montré que le manque de mouvement de ces stimuli pouvaient être un obstacle à la réaction des animaux qu'on étudiait, car ils pouvaient les considérer de manière rapide comme non-vivant et donc ne pas prêter attention aux autres stimuli qui sur leurs congénères permettent de les reconnaître comme conspécifiques (Pongrácz et Altbäcker, 2000).

C'est pourquoi Kubinyi et ses collègues (Kubinyi et al., 2004) ont proposé, dans le cadre de l'étude de la reconnaissance de conspécifiques chez les chiens, d'utiliser un robot AIBO dont la forme et la taille ressemble à celle de certaines espèces de chiens, et dont les programmes de mouvements sont inspirés de modèles comportementaux canins (Arkin et al., 2001). Ils ont ainsi réalisé des expériences systématiques en comparant la réaction de chiens d'âges et de sexes variés, en interaction avec quatre types de partenaires : un chien jeune de la taille du robot AIBO, un robot AIBO standard, un robot AIBO auquel on a mis une fourrure imprégnée de l'odeur des chiens, et une voiture télécommandée. Ces interactions ont eu lieu dans deux situations différentes : interactions neutres et libres dans lesquelles le chien et son partenaire sont dans la même pièce avec des humains, et interactions en situation de compétition potentielle dans laquelle un bol de nourriture est donné au chien testé et vers lequel se dirige le partenaire (les robots et la voiture téléguidées sont programmés pour se diriger vers la nourriture). A chaque fois, les expérimentateurs notaient précisément le comportement du chien étudié, incluant les temps d'approche ou d'éloignement par rapport au partenaire, les temps de regards vers le partenaire, le nombre de fois ou le chien vient sentir le partenaire, et le nombre de fois où le chien aboie ou grogne. Une fois ces expériences terminées, les chercheurs ont ainsi pu montrer que le robot AIBO doté de fourrure odorante était inspecté par tous les chiens et dans toutes les situations autant que le vrai jeune chien et significativement plus que le robot AIBO sans fourrure et la voiture téléguidée. Ils ont aussi 
montré une différence importante entre les chiens adultes et juvéniles dans la situation de compétition : alors que la plupart des chiens adultes ignoraient les robots et la voiture pendant qu'ils se nourrissaient, la plupart des jeunes chiens grognaient vers les robots et la voiture quand ils s'approchaient de leur gamelle. Enfin, en comparant les résultats de ces expériences avec les expériences antérieures utilisant des maquettes ou des photos, ils ont montré que l'utilisation de robots permettait de déclencher un nombre significativement supérieur de réaction chez les chiens. Cela montre plusieurs choses : d'abord, l'utilisation de robots permet de faire des expériences à la fois plus contrôlables que l'utilisation de partenaires naturels (conspécifiques, animaux d'une autre espèce) et plus sophistiquées que l'utilisation de photos ou de maquettes. Ensuite, le mouvement quadrupède combiné à une empreinte olfactive appropriée attire chez le chien un attention comparable à celle qu'il a auprès d'êtres vivants (mais pas nécessairement conspécifiques). Enfin, cette expérience fournit des indices appuyant la thèse selon laquelle la reconnaissance des conspécifiques est une capacité qui se développe chez les chiens : les juvéniles semblent en avoir une conception très approximative. On voit donc comment l'utilisation d'un robot peut permettre de faire progresser la compréhension de la manière dont les chiens distinguent les êtres animés des objets inanimés et apprennent à reconnaître leurs conspécifiques.

Robots et études de l'autisme chez les enfants humains. Les robots ne sont pas seulement utilisés comme dispositif d'étude expérimental du comportement des insectes ou des mammifères comme les chiens, mais aussi pour l'étude de l'homme. En particulier, ce sont développés ces dernières années plusieurs groupes de recherche mettant en avant l'intérêt d'utiliser des robots pour étudier, diagnostiquer et même participer à la thérapie des enfants atteints d'autisme (Dautenhahn et al., 2003 ; Scassellati, 2005 ; Duquette et al., 2008 ; Kozima et al., 2005 ; Stanton et al., 2008). L'autisme est un problème dans le développement de l'enfant qui est caractérisé par un certain nombre de symptômes (Volkmar et al., 2004) : 1) déficit d'interactions sociales : les enfants autistes concentrent beaucoup plus leur attention sur les objets que sur les personnes qui les entourent, ne sont pas proactifs socialement et ont de grandes difficultés pour interpréter le comportement des autres; 2) problèmes de communication: ces enfants ont souvent des problèmes de langage, se manifestant par exemple par des choix inadaptés d'intonation ou de mots ; 3) présence de comportements répétitifs et aversion pour la nouveauté : les enfants autistes ont tendance à s'enfermer dans des rituels et craignent les situations qui dévient de leur routine.

Diagnostiquer l'autisme. Un des premiers obstacles qui se posent aux scientifiques et au médecin est celui du diagnostique (Scassellati, 2005). En effet, l'autisme recouvre en fait un large spectre de problèmes développementaux et sa spécification est comportementale (Volkmar et al., 2004) : il n'y a pas de test sanguins, génétiques ou même de marqueurs nets et visibles en imagerie cérébrale. Cela s'explique d'une part par le fait qu'il semble que le spectre de ces problèmes développementaux est probablement dû à un ensemble varié de causes que la communauté scientifique est loin d'avoir identifiées. L'obtention de données statistiques et fiables concernant les populations d'enfants autistes est un élément essentiel pour progresser sur notre compréhension de leurs déficits. Or, cela nécessite des protocoles de diagnostique et d'évaluation précis et le plus objectifs possibles. Malheureusement, et malgré l'existence de protocoles standardisés (e.g. Mullen, 1995), ceux-ci reposent sur l'appréciation intuitive des cliniciens sur les capacités sociales de l'enfant et relatives au contrôle de leur regard, leurs expressions faciales, ou leur gestes. On observe ainsi pour un certain nombre de cas des désaccords entre cliniciens, d'autant plus exacerbé que certains enfants refusent d'interagir avec eux. 
Scassellati a proposé et expérimenté l'utilisation de robots pour améliorer la qualité des diagnostiques, ainsi que le suivi de l'évolution des capacités sociales et cognitives des enfants autistes (Scassellati, 2005). Cette idée repose d'abord sur une observation faite par tous les groupes de recherche cités ci-dessus : les enfants autistes sont très fortement motivés par les interactions avec des robots, plus qu'avec des jouets habituels, et même les enfants refusant d'interagir avec les cliniciens ou les thérapeutes s'engagent dans des interactions avec le robot en leur présence (Dautenhahn et al., 2003 ; Scassellati, 2005 ; Stanton et al., 2008). Ensuite, il est possible de programmer les robots pour qu'ils soient en même temps interactifs mais produisant des stimuli réellement standardisés et répétables permettant de disposer d'un même test pour tous les enfants. Ceci est couplé avec l'utilisation d'un ensemble de méthodes d'enregistrements et d'analyses des situations sociales développées par la communauté de robotique sociale (e.g. Breazeal, Robinson-Mosher et Scassellati, 2004), permettant de mesurer précisément et en temps réel des éléments comme la direction du regard, la position dans l'environnement et par rapport aux personnes et aux objets en particuliers, ou les caractéristiques de l'intonation de la voix, qui sont justement des éléments pertinents pour le diagnostique et le suivi de l'autisme. En outre, ces robots pouvant être de forme et de fabrication relativement simple, il est possible de les faire sortir des institutions hospitalières afin que les enfants puissent continuer à jouer avec chez eux. Si cela était réalisé à grande échelle et systématiquement, des données beaucoup plus précises et naturelles sur chaque enfant pourraient être obtenues, ce qui permettrait d'adapter et de suivre beaucoup plus efficacement les méthodes thérapeutiques. Cela permettrait aussi d'obtenir des données statistiques sur les populations de ces enfants, ouvrant des portes importantes pour faire avancer la compréhension que les scientifiques ont de ce spectre de problèmes développementaux (Scassellati, 2005).

L'apport thérapeutique des robots. Outre les apports potentiels de l'utilisation des robots avec des enfants autistes pour le diagnostique, ces équipes de recherche ont également montré qu'ils constituaient un potentiel thérapeutique à la fois grand et inattendu (Dautenhahn et al., 2003 ; Scassellati, 2005 ; Duquette et al., 2008 ; Kozima et al., 2005 ; Stanton et al., 2008). Comme expliqué plus haut, les robots provoquent un intérêt fort chez les enfants autistes : très souvent ceux-ci s'engagent avec les robots dans une interaction faisant intervenir spontanément imitation gestuelle et vocale, sourires, jeu libre, et arrêt des comportements stéréotypés, ce que l'on observe beaucoup plus rarement quand ils interagissent avec des partenaires humains où des jouets plus simples (Werry et al., 2001). L'une des raisons principales identifiée est que ces enfants ont de grandes difficultés à faire sens des comportements vocaux, gestuels et faciaux des adultes et des autres enfants à cause de leur complexité et de leur variabilité. Par exemple pour les expressions faciales des émotions, il semble qu'ils ne parviennent pas à distinguer, dans la masse des indices musculaires du visage, ceux qui sont caractéristique d'une émotion de ceux qui ne le sont pas et résultent de la variabilité naturelle des mouvements du visage. Cette incompréhension provoque chez eux un retrait et un blocage psychologique qui accentue avec le temps leurs déficits en termes de savoir-faire sociaux. C'est aussi une des raisons pour lesquelles ils se tournent vers des comportements stéréotypés mettant en jeu la manipulation répétitive d'objets physiques ou la génération de gestes rituels. Les robots apparaissent dans ce paysage comme comblant l'écart entre les objets physiques et les partenaires humains. En effet, du fait qu'ils sont autopropulsés, en apparence autonome, et souvent dotés de formes anthropoïdes ou animaloïdes (Wada et al., 2002 ; Dautenhahn et Billard, 2002 ; Kozima et Nakagawa, 2006, Duquette et al., 2008), les enfants leurs attribuent facilement un caractère d'êtres animés dotés d'intentions (Premack, 1990) : ils sont donc considérés comme des partenaires sociaux 
potentiels et attirent leur attention. Cependant, au contraire des partenaires sociaux humains, le comportement des robots peut être simple et très prédictible, donc rassurant et permettant aux enfants de s'engager dans une interaction positive plutôt que de se poser en retrait comme avec les autres humains. Cela a tout d'abord l'immense avantage de permettre à ces enfants de reprendre un peu de confiance dans leurs comportements sociaux (Dautenhahn et al., 2003). Ensuite, grâce au fait que les robots peuvent être adaptés - forme et comportement - à chaque enfant (c'est le centre de projets de recherche comme IROMEC, http://www.iromec.org), le cadre de ces interactions positives peut permettre aux thérapeutes de les faire travailler des capacités sociales spécifiques à leurs problèmes développementaux : par exemple l'imitation gestuelle (Dautenhahn et Billard, 2002), l'imitiation faciale (Nadel et al., 2004), la prise de tour de jeu (Dautenhahn et Werry, 2004), la conscience de leur corps (Dautenhahn et al. , 2003), où l'attention partagée (Duquette et al., 2008). Enfin, un certain nombre d'expériences récentes ont démontré que l'utilisation des robots avec ces enfants ne permettait pas seulement d'établir et de permettre la pratique d'interaction sociales fondatrices entre l'enfant et le robot, mais également de provoquer des interactions sociales inhabituellement riches entre l'enfant et d'autres partenaires humains présents avec lui dans la pièce où est le robot. Par exemple, (Duquette et al., 2008) ont montré que l'utilisation d'un robot pouvait permettre d'établir une attention partagée accrue (contact visuel, proximité physique) et des imitations de sourires entre des enfants autistes confrontés ensemble à un même robot. (Robins et Dautenhahn, 2007) ont quant à eux montré que l'utilisation d'un robot pouvait permettre au thérapeute d'installer une relation avec l'enfant beaucoup plus positive. Les robots semblent donc pouvoir jouer un rôle de médiateur entre les enfants autistes et les autres humains, et donc participer à l'apprentissage de savoir-faire sociaux pour une meilleure intégration dans notre société.

\section{Conclusion}

Un siècle après l'exemple précurseur de l'« electric dog » de Hammond et Miessner, les interactions entre la robotique et les sciences du vivant se sont donc multipliées. En particulier, outre l'impact que la biologie peut avoir sur la manière dont les ingénieurs construisent les robots, sur lequel nous ne nous sommes pas attardés ici car il est abondamment décrit dans la littérature (voir Arkin, 1998 par exemple), nous avons vu à quel point la construction et l'utilisation de robots pouvait elle-même transformer la manière dont nous pouvons penser le vivant. Comme nous l'avons décrit, cela est particulièrement vrai pour les sciences de l'esprit et du comportement. Nous avons d'abord vu comment l'implémentation de théories comportementales spécifiques, à l'instar du travail de Hammond et Miessner, pouvait permettre de rendre plausible, ou au contraire d'infirmer, leur plausibilité et leur cohérence interne. Nous avons vu que des expériences robotiques pouvaient permettre d'établir des conditions suffisantes, ou des preuves de non-nécessité de la présence de certains mécanismes, pour rendre compte de certains comportements, et que ceci pouvait avoir un rôle structurant dans le débat scientifique. Plus encore que l'évaluation d'hypothèses existantes, certains travaux en robotique ont également permis d'une part d'articuler et de développer les intuitions des scientifiques sur des concepts nouveaux comme le rôle de la morphologie dans l'intelligence ou l'auto-organisation du langage, et d'autre part de générer de nouvelles hypothèses, comme celles relatives aux dynamiques culturelles Darwinienne des langues et leur impact sur leur apprenabilité. Nous avons également vu que les robots pouvaient aussi se révéler des outils précieux pour la mise en place de dispositifs expérimentaux permettant d'étudier de manière plus systématique les propriétés du comportement des animaux et de la psychologie humaine. Pour finir, un certain nombre de travaux récents suggèrent que les 
robots pourraient être aussi des outils thérapeutiques uniques pour tenter de remédier aux troubles du développement cognitif comme l'autisme.

Ainsi, les robots ne sont pas seulement des prismes au travers desquels l'homme peut comprendre le vivant d'une manière nouvelle et complémentaire des méthodes traditionnelles, mais ils semblent pouvoir jouer le rôle de médiateurs permettant à certains humains atteints de problèmes cognitifs de se rapprocher des autres hommes. Les robots, bien qu'ils soient encore dénués de la complexité de l'esprit et du comportement de la plupart des êtres vivants, ont donc un rôle essentiel à jouer dans les sciences de l'esprit et du comportement.

\section{Références}

Andry, P., Gaussier P., Moga S., Banquet J.P., Nadel J. (2001) Learning and communication in imitation : an autonomous robot perspective, IEEE Transactions on Systems, Man and Cybernetics, Part A : Systems and Humans, 31(5), pp. 431-444.

Arkin, R. (1992) Behavior-based robot navigation in extended domains, Journal of Adaptive Behavior, 1(2), pp. 201-225.

Arkin, R. (1998) Behavior-Based Robotics, The MIT Press, Cambridge, MA.

Arkin, R.C., Fujita, M., Takagi T., Hasegawa, R. (2001) Ethological Modeling and Architecture for an Entertainment Robot. ICRA 2001: 453-458.

Ashby, W.R. (1940) Adaptiveness and equilibrium, Journal of Mental Science, 86 : 478-483.

Ball, P. (2001)The self-made tapestry, Pattern formation in nature, Oxford University Press.

Boden, M.A. (2006) Mind as machine : a history of cognitive science, Oxford University Press, Oxford, UK.

Boe, L.J., Schwartz J.L. et Vallee N. (1995) The prediction of vowel systems perceptual contrast and stability In: K. E, Editors, Fundamentals of Speech Synthesis and Recognition, Wiley, Chichester, pp. 185-213.

de Boer, B. (2001) The origins of vowel systems, Oxford Linguistics, Oxford University Press.

Breazeal, C. (2002) Designing Sociable Robots, MIT Press.

Brooks, R.A. (1991) Intelligence without representation. Artificial Intelligence, 47, 139-160.

Camazine, S., Deneubourg, J-L., Franks, N.R., Sneyd, J., Theraulaz, G., Bonabeau E. (2001) Self-Organization in Biological Systems, Princeton Univ Press.

Chomsky, N. (1957) Syntactic Structures. The Hague: Mouton. Reprint. Berlin and New York (1985). 
Coleman, M., Ruina, A. (1998) An Uncontrolled Toy That Can Walk But Cannot Stand Still. Physical Review Letters April 1998, Vol 80, Issue 16 pp. 3658 - 3661.

Colgan, P.W. (1983) Comparative Social Recognition. Wiley-Interscience Publication.

Cordeschi, R. (2002) The Discovery of the Artificial: Behavior, Mind and Machines Before an Beyond Cybernetics. Kluwer Academic Publishers, Dordrecht.

Dautenhahn, K., et Billard, A. (2002) Games children with autism can play with Robota, a humanoid robotic doll. In Proceedings of the Cambridge workshop on universal access and assistive technology, pp. 179-190, New York: Springer.

Dautenhahn, K., Werry, I., Salter, T., and Boekhorst, R. t. (2003) Towards adaptive autonomous robots in autism therapy: Varieties of interactions. In Proceedings of the IEEE International Symposium on Computational Intelligence in Robotics and Automation, Kobe. Piscataway, NJ: IEEE, 2003, 577-582.

Dautenhahn, K., \& Werry, I. (2004) Towards interactive robots in autism therapy: Background, motivation and challenges. Pragmatics and Cognition, 12(1), 1-35.

Dupuy, J-P. (2005) Aux origines des sciences cognitives, La Découverte, Paris.

Duquette, A., Michaud, F., Mercier, H. (2008) Exploring the use of a mobile robot as an imitation agent with children with low-functioning autism, Autonomous Robot, 24, pp. 147157.

Dreyfus,H. et Dreyfus, S. (1982) Mind Over Machine, Free Press.

Dreyfus, H. (1972) What Computers Can't Do: A Critique of Artificial Reason. New York: Harper \& Row.

Driesch, H. (1909) Philosophie des Organischen, Engellman, Leipzig.

Egelhaaf, M., Borst, A. \& Reichardt, W. (1989) The nonlinear mechanism of direction selectivity in the fly motion detection system. Naturwissenschaften, 76, 32-35.

Ernst G.W., Newell A. (1969) GPS: a case study on generality and problem solving, New York, Academic Press.

Exner, S. (1891) The Physiology of the Compound Eyes of Insects and Crustaceans, Translated by R.C Hardie, Springer, Berlin, pp. 130-131.

Franceschini, N., Ruffier, F., Serres J. (2007) A bio-inspired flying robot sheds light on insect piloting abilities Current biology Volume 17, Issue 4, 20 February 2007, Pages 329-335

Götz, K.G., Wenking, H. (1973) Visual control of locomotion in the walking fruitfly drosophila, Journal of Comparative Physiology, 85 :235-266.

Guillaume, P. (1925) L'imitation chez l'enfant, Paris : Alcan. 
Hirose, M., Haikawa, Y., Takenaka, T., Himi, K. (2001) Development of Humanoid Robot ASIMO, Proc. IEEE/RSJ Int. Conference on Intelligent Robots and Systems, Workshop 2.

Huber, S. A., \& Bulthoff, H. H. (1998) Simulation and robot implementation of visual orientation behaviour of flies. in Pfeifer, R., Blumberg, B., Meyer, J. A., and Wilson, S. W. (editors)From animals to animats 5 (pp. 77-85). Cambridge, Mass.: MIT Press.

Hurford, J., Studdert-Kennedy, M., and Knight, C. (1998) Approaches to the Evolution of Language: Social and Cognitive Bases. Cambridge University Press.

Hurford, J. (2001) Random Boolean Nets and Features of Language. IEEE Transactions on Evolutionary Computation, 5(2):111--116.

Kaplan, F. (2001) La naissance d'une langue chez les robots. Hermes Science, Paris

Kaplan, F. et Oudeyer P-Y. (à paraître) Le corps comme variable expérimentale, Revue Philosophique de la France et de l'étranger, Presses Universitaires de France.

Kauffman, S. (1996) At Home in the Universe: The Search for Laws of Self-Organization and Complexity, Oxford University Press.

Kirby, S. (2001) Spontaneous evolution of linguistic structure - an iterated learning model of the emergence of regularity and irregularity, IEEE Trans. Evol. Comput. 5 (2), pp. 102-110.

Kozima, H., Nakagawa, C., and Yasuda, Y. (2005) Designing and observing human-robot interactions for the study of social development and its disorders. In Proceedings of the 2005 IEEE International Symposium on Computational Intelligence in Robotics and Automation, Espoo, Finland. Piscataway, NJ: IEEE, 2005, 41-46.

Kozima, H., Nakagawa. C. (2006) Interactive robots as facilitators of children's social development. Mobile Robots: Toward New Applications, pp. 269-286.

Kubinyi, E. Miklosi, A. Kaplan, F. Gacsi, M, Topal, J. andi Csanyi, V. (2004) Social behaviour of dogs encountering AIBO, an animal-like robot in a neutral and in a feeding situation, Behavioural Processes, Vol 65/3 pp 231-239.

Lack, D. (1939) The behaviour of the robin: I and II. Proc. Zool. Soc. Lond. A. 109, 169-178.

Liljencrantz, J. et Lindblom, B. (1972) Numerical simulation of vowel quality systems: the role of perceptual contrast. Language 48: 839-862.

Lindblom, B., MacNeilage, P., and Studdert-Kennedy, M. (1984) Self-organizing processes and the explanation of language universals. In Butterworth, B. and Bernard, C. and Dahl, O., editors, Explanations for Language Universals, pages 181--203.

Loeb, J. (1900) Comparative physiology of the brain and comparative psychology, Putnam, New-York. 
Loeb, J. (1918) Forced movements, tropisms, and animal conduct, Lippincott, Philadelphia and London.

MacKenzie, D. and Balch T. (1993) Making a Clean Sweep: Behavior-based Vacuuming, Working notes of 1993 AAAI Fall Symposium : Instantiating real-world agents, AAAI, Raleigh, N.C.

Maris, M. and Boekhorst, R te. (1996) Exploiting Physical Constraints: Heap formation through behavioral error in a group of robots. In proc. of IROS '96, Nov. 4-8, Osaka, Japan

Maturana, H., Varela, F. (1980) Autopoiesis and Cognition: The Realization of the Living. Boston: Reidel.

Mc Carthy, J., Minsky, M., Rochester, N., Shannon, C. (1955) A proposal for the Darmouth summer research project on artificial intelligence,

http://www.formal.stanford.edu/jmc/history/dartmouth/dartmouth.html

McDougall, W. (1911) Body and mind, a history and a defense of animism, Methuen, London.

McGeer, T. (1990) Passive walking with knees, In: Proc. 1990 IEEE Robotics \& Automation Conference, Cincinnati, OH, pp. 1640-1645.

McGeer, T. (1993) Dynamics and control of bipedal locomotion. J. Theoretical Biology 163 , 277-314

Mehler, J., Christophe, A., Ramus, F. (2000) What we know about the initial state for language. In: Marantz, A., Miyashita, Y., O'Neil, W. (Eds.), Image, Language, Brain: Papers from the ${ }^{-}$rst Mind-Brain Articulation Project symposium. Cambridge, MA: MIT Press, pp. $51--75$.

Merleau-Ponty, M. (1942) La structure du comportement, Paris, Press Univeristaire.

Merleau-Ponty, M. (1962) Phenomenology of Perception,, Routledge \& Kegan Paul.

Michelsen, A., Andersen, B., Storm J., Kirchner, W.H., Lindauer, M. (1992) How honeybees perceive communication dances, studied by means of a mechanical model. Behavioral Ecology and Sociobiology, 30, 143-150.

Miessner, B.F. (1912) The wirelessly directed torpedo. Some new experiments in an old field, Scientific American, June : 53.

Mullen, E.M. (1995) Mullen scales of early learning, AGS Edition, Circle Pines, MN : American Guidance Service.

Nadel, J., Revel, A., Andy, P., Gaussier, Ph. (2004). Toward communication, first imitations in infants, low-functioning children with autism and robots. Interaction Studies, 5(1), 45-74.

Newell, A., Simon, H.A. (1976) Computer science as empirical enquiry : symbols and search, Communications of the ACM, 19(3), pp. 113-126. 
Oudeyer, P-Y (2005a) How phonological structures can be culturally selected for learnability, Adaptive Behavior, 13(4), pp. 269--280.

Oudeyer P-Y (2005b) The self-organization of speech sounds, J Theor Biol 233(3):435-449

Oudeyer, P-Y. (2006) Self-Organization in the Evolution of Speech, Studies in the Evolution of Language, Oxford University Press.

Oudeyer, P-Y. and Kaplan, F. (2007) Language Evolution as a Darwinian Process:

Computational Studies, Cognitive Processing, 8(1), pp. 21--35.

Pfeifer, R. and Scheier, C. (1999) Understanding Intelligence. The MIT Press, Cambdrige, MA.

Pinker, S. et Bloom P. (1990) Natural Language and Natural Selection. In: Behavioral and Brain Sciences 13.4: 707-726.

Pongrácz, P., Altbäcker, V. (2000) Ontogeny of the responses of European rabbits (Oryctolagus cuniculus) to aerial and ground predators. Can. J. Zool. 78, 655-665. Premack, D. (1990). The infant's theory of self-propelled objects. Cognition, 26, 1-16.

Putnam, H. (1975) Mind, Language and Reality. Philosophical Papers, vol. 2. Cambridge: Cambridge University Press, 1975.

Robins, B., Dautenhahn, K. (2007) Encouraging social interaction skills in children with autism playing with robots : a case study evaluation of triadic interactions involving children with autism, other people (peers and adults) and a robotic toy, Enfance, 1, pp. $72-81$.

Robinson-Mosher, A., Scassellati, B. (2004) Prosody recognition in male infant-directed speech. Proceedings of the 2004 IEEE/RSJ International Conference on Intelligent Robots and Systems (IROS), Sendai, Japan.

Samuel , A. (1959) Some Studies in Machine Learning Using the Game of Checkers. IBM Journal 3 (3): 210-229.

Santos-Victor, J., Sandini, G., Curotto, F. \& Garibaldi, S. (1995) Divergent stereo in autonomous navigation: from bees to robots. International Journal of Computer Vision, 14, $159-177$.

Scassellati, B. (2005) Using robots to study abnormal social development. Proceedings of the Fifth International Workshop on Epigenetic Robotics (EpiRob). Nara, Japan.

Schwartz J.L., Boe L.J., Valle N. and Abry C. (1997) Major trends in vowel systems inventories, J. Phonetics 25, pp. 255-286.

Srinivasan, M.V., Lehrer, M., Kirchner, W.H., Zhang, S.W. (1991) Range perception through apparent image speed in freely flying honeybee, Visual Neuroscience, $6: 519-535$. 
Srinivasan, M. V., Chahl, J. S., Weber, K. \& Venkatesh, S. (1999) Robot navigation inspired by

principles of insect vision. Robotics and Autonomous Systems, 26, 203-216.

Stanton, C. M., Kahn, P. H., Jr., Severson, R. L., Ruckert, J. H., \& Gill, B. T. (2008). Robotic animals might aid in the social development of children with autism. Proceedings of the 3rd ACM/IEEE International Conference on Human-Robot Interaction (HRI 2008) (Amsterdam, The Netherlands, March 12-15, 2008). Piscataway, NJ: Institute of Electrical and Electronics Engineers (IEEE).

Steels, L. (1995) A self-organizing spatial vocabulary. Artificial Life, 2(3):319--332.

Steels, L. (1997) The synthetic modeling of language origins. Evolution of Communication 1.

Steels, L., Kaplan, F. (2002) Bootstrapping grounded word semantics. In: Briscoe T (ed)

Linguistic evolution through

language acquisition: formal and computational models. Cambridge University Press, Cambridge, pp 53-74

Steels, L. (2003) Evolving grounded communication for robots. Trends Cogn Sci 7(7):308312

Steels, L. (2005) The Emergence and Evolution of Linguistic Structure: From Lexical to Grammatical Communication Systems. Connection Science, 17(3-4):213-230 .

Stevens, K. (1972) The Quantal nature of speech: evidence from articulatory-acoustic data. Mc Graw-Hill, New York, pp. 51-66.

Thompson, D. (1917) On Growth and Form, Reprint, Cambridge University Press (2000).

Tomasello, M. et Carpenter, M. (2007) Shared Intentionality, Developmental Science, $10: 1$, pp. $121-125$.

Triantafyllou, M. S. et Triantafyllou, G. S. (1995). An efficient swimming machine. Scientific American, 272:40-48.

Turing, A.M. (1952) The Chemical Basis of Morphogenesis. Philosophical Transactions of the Royal Society of London, volume B 237, pages 37--72.

Volkmar, F.R., Lord, C., Bailey, A., Schultz, R.T., Klin, A. (2004) Autism and pervasive developmental disorders. Journal of Child Psychology and Psychiatry, 45(1), 1-36.

Von Foerster, H. (1958) Basic Concepts of Homeostasis." In: Homeostatic Mechanisms, Upton, New York, pp. 216-242.

Von Frisch, K. (1971) Bees - Their vision, chemical senses, and language. Revised Edition. Cornell University Press, Ithaca and London. 
Wada K., Shibata T., Saito T., and Tanie K. (2002) Analysis of factors that bring mental effects to elderly people in robot assisted activity. In Proc. IEEE/RSJ International

Conference on Intelligent Robots and Systems (IROS 2002), pages 1152--1157. IEEE Press.

Walter, W.G. (1951) A machine that learns, Scientific American, $185: 60-63$.

Wallace, G.K. (1959) Visual scanning in the desert locust schistocerca gregaria, Journal of Experimental Biology 36, pp. 512-525.

Webb, B. (2000) What does robotics offer animal behaviour? Animal Behaviour 60:545--558

Wenner, A. and Wells, P.H. (1990) Anatomy of a controversy - the question of a « language » among bees. Columbia University Press, New York.

Werry, I., Dautenhahn, K., \& Harwin, W. (2001) Evaluating the response of children with autism to a robot. In Proceedings of the rehabilitation engineering and assistive technology society of North America (RESNA).

Zuidema, W. (2003) How the poverty of the stimulus solves the poverty of the stimulus. In: Becker S, Obermayer K (eds)

Advances in Neural Information Processing 15. MIT Press, Cambridge, pp 51-68 